\title{
POSTTRAUMATIC PNEUMOPERICARDIUM: A SIGN OF SEVERE INJURY OR RADIODIAGNOSTIC RARITY?
}

\author{
Vladimír Lonskýl, Jiř̌ Mandảk ${ }^{1}$, Jan Harrer ${ }^{1}$, Martin Tuna ${ }^{1}$, Petr Dvoráa ${ }^{2}$, Tomáš Dědek ${ }^{3}$, Jan Dominik ${ }^{1}$ \\ Charles University in Prague, Faculty of Medicine and University Hospital in Hradec Králové, Czech Republic: \\ Departments of Cardiac Surgery ${ }^{1}$, Radiology ${ }^{2}$ and Traumatology ${ }^{3}$
}

Summary: We present three cases of pneumopericardium following blunt chest trauma injury. All three patients were victims of road traffic accidents. All had multiple associated injuries and pneumopericardium was found as the additional finding. Pneumopericardium was treated conservatively with thoracic drains placement and patients observation. Transesophageal echocardiography was used as a method of choice for exclusion of cardiac air tamponade. All three patients survived.

Key words: Blunt chest trauma; Pneumopericardium; Transesophageal echocardiography

\section{Introduction}

Cardiac surgeons are sometimes asked for assistance at the emergency departments to help in making diagnosis in chest trauma patients. During one month we met with 3 cases of pneumopericardium (PNP) in patients involved in motor vehicle crashes resulting in severe blunt chest trauma. The pneumopericardium was found as the additional finding in all 3 patients and we were asked to decide whether it is a serious complication or not.

Posttraumatic pneumopericardium in general is a very rare complication and only several cases have been published in the world literature (17). Their exact management it is also unclear. These were the reasons for presenting these three cases.

\section{Case reports}

Case 1

A 18-year old man has been admitted to the trauma department of our hospital after a high speed vehicle crash. The patient was GCS 8 , intubated on site due to shortness of breath and oximetric saturation of $81 \%$. Transported by air rescue service. At admission thoracic and abdominal CT scanning showed bilateral pneumothorax with a tension on the right side, pneumopericardium up to $20 \mathrm{~mm}$ of pericardial separation (Fig. 1), and contusion of the right lung, and perisplenic hematoma. In addition right humerus and Th 12-L1 vertebrae fractures were observed without neurological symptomes. Bilateral chest tubes were inserted and transesophageal echocardiography was performed as the first steps. No pericardial effusion, normal cardiac contractions without any signs of tamponade, normal valvular apparatus and normal descending thoracic aorta were found. After it splenectomy and humerus fracture external

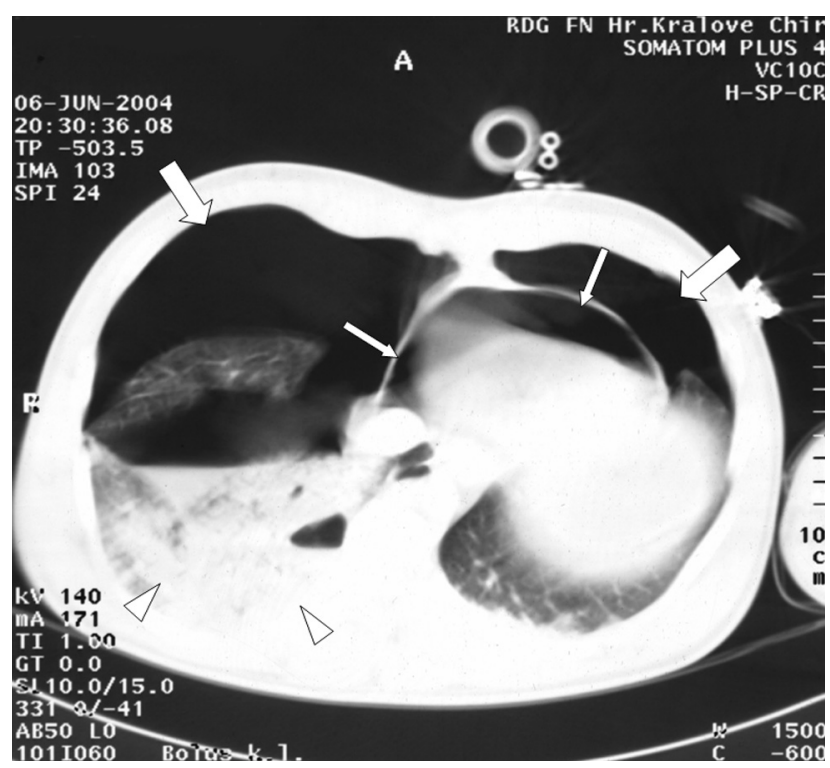

Fig. 1: 18-year old man who was injured in a high-speed motor vehicle accident. Admission thoracic CT scan shows bilateral pneumothorax. (thick arrows) On the right side is tension pneumothorax with mediastinal deviation to the contralateral side. Air in pericardium in front of the heart up to $20 \mathrm{~mm}$ of separation (thin arrows). In right lower lobe of the lung is seen area of contusion. (arrowheads). 
osteosysthesis were perfomed. Ten days after admission the last chest tube was removed. The pneumopericardium had completely dissapeared on chest radiography. Three days later tracheostomy cannula was removed. Fifteen days after admission the final humerus osteosynthesis was performed and 33 days after admission the patient was discharged from trauma center to a peripheral hospital.
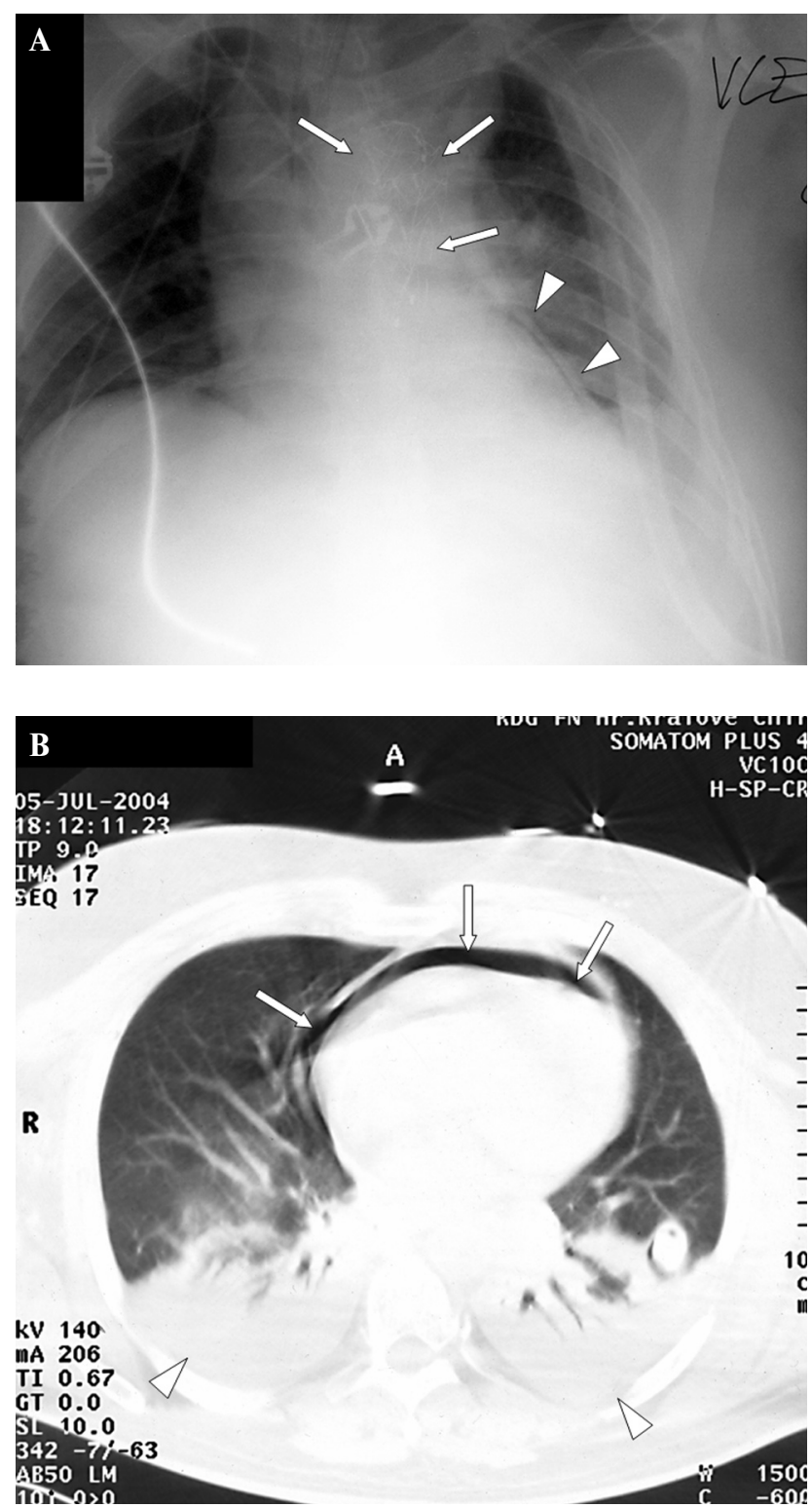

Fig. 2: A 42-year old man after aortic stentgraft implantation and left thoracotomy, splenectomy and diaphragm reconstruction due to multiple organ injury.

A. AP chest radiograph shows gas within the pericardial space outlining left ventricle. (arrowheads). Implanted stentgraft. (arrows).

B. On Thoracic CT scan is seen large bilateral lung contusion (arrowheads) and pneumopericardium up to $18 \mathrm{~mm}$ of the pericardium separation (arrows).
Case 2

A 42-year old man was admitted to the trauma department of our hospital after a high speed vehicle crash as a front seat passenger. The patient was GCS 14, intubated on site and transported by the air rescue service. At admission he was hemodynamically stable. Thoracic and abdominal CT scanning showed a large mediastinal hematoma, transsection of the thoracic aorta, left diaphragm rupture with gastric and lacerated spleen herniation into the left thoracic cavity and bilateral lung contusion. In addition left tibia fracture was observed.

The stentgraft placement into ruptured thoracic aorta was performed as the first step. Secondary left thoracotomy, splenectomy and diaphragm reconstruction and tibia osteosyntesis were done. On the second day another CT examination was done to check the stentgraft position and the thoracic cavities. No signs of bleeding but the pneumopericardium up to $18 \mathrm{~mm}$ of the pericardium separation was found (Fig. 2A,B). At this time the patient was tachycardic to 160 beats/min and hypertensive $(180 \mathrm{mmHg})$. On the basis of these findings we decided to perform transesophageal echocardiography to eliminate the cardiac air tamponade. The EF was $65 \%$ with normal heart contractions, without any signs of cardiac tamponade, with optimal position of the stentgraft. That's why conservative treat-

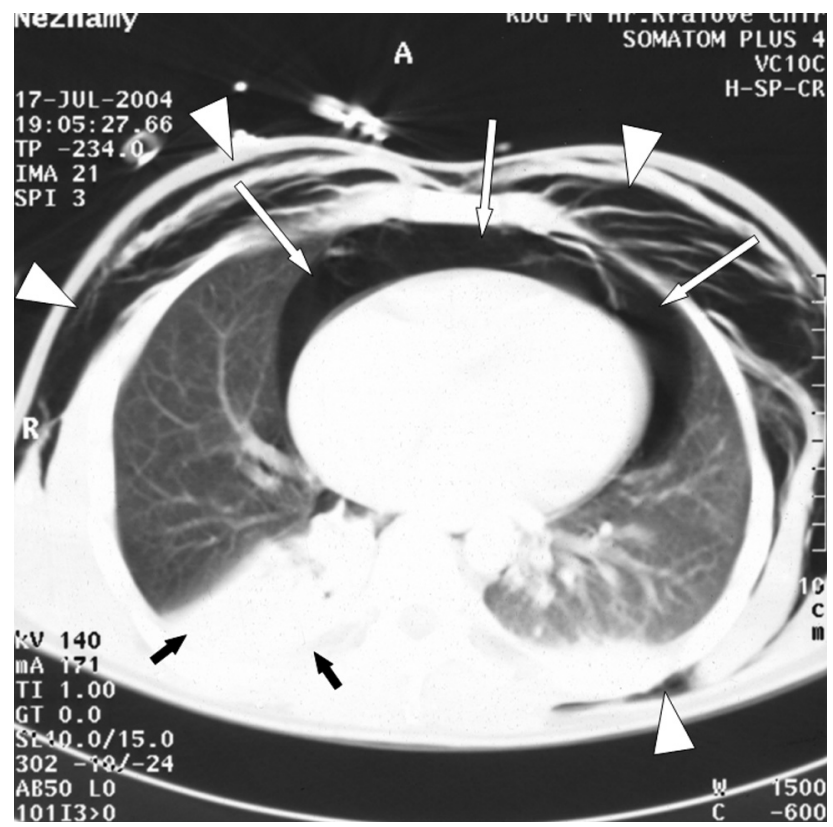

Fig. 3: A 22-year old who was injured in a high-speed motorcycle crash.

Thoracic CT after splenectomy shows large subcutaneous emphysema (arrowheads), pneumomediastinum and pneumopericardium up to $20 \mathrm{~mm}$ of the pericardium separation (arrows) and consolidation in the right lower lobe due to contusion. (black arrows). 
ment with patient's observation was chosen and pneumopericardium disappeared spontaneously within a few days.

After 22 days of artificial ventilation complicated with bronchopneumonia and pleural effusions the patient was weaned from ventilator. After rehabilitation 52 days after admission he was discharged home.

\section{Case 3}

A 22-year old man was admited to the trauma department of our hospital after a high speed motorcycle crash. Having suffered from the chest pain and complaining of shortness of breath he was intubated on site, mechanically ventilated and transported by ambulance. On arrival the patient was in critical status with an enormously massive subcutaneous emphysema of face, chest and abdominal wall. Urgent bilateral chest drainage was performed without any scanning and right tension pneumothorax was detected without intrathoracic bleeding. After that circulation got stable and because of high suspicion of intraabdominal bleeding after abdominal ECHO having been performed the patient was taken to OR. Due to spleen laceration splenectomy was performed. Thoracic CT scan was done after the operation. Multiple bilateral rib fractures, subcutaneous emphysema, pneumomediastinum and pneumopericardium up to $20 \mathrm{~mm}$ of the pericardium separation were found (Fig. 3). Bilateral thoracic drains were in good position without presence of pneumothorax and the signs of intrathoracic bleeding. The patient was stable without symptoms of cardiac tamponade. Despite of it the TEE was performed to check cardiac status. The EF was 70\% without any signs of cardiac tamponade. Conservative approach had been chosen and after ten days the second CT scan did not show any sign of pneumopericardium.

The patient was extubated on the third day and transferred to the regional hospital eleven days after admission.

\section{Discussion}

The identification of pneumopericardium by chest radiography was described by Wenckebach in 1910 and elaborated upon by Cimmiro more recently $(3,12)$.

Pneumopericardium secondary to a blunt chest trauma is generally due to 1 of 3 mechanisms $(6,8)$ :

1) Pneumothorax with pleuropericardial tear. An abrupt increase of intrathoracic and intraalveolar pressure may lead to a disruption of the alveoli. If there is an associated tear in the visceral lung pleura, then air escapes into the thoracic cavity. A similar tear in the pericardium could allow air to escape into the pericardial sac. Pericardial tissue may act as a shutter valve, letting air into the sac but not back out into the thoracic cavity. Than the tension pneumopericardium can occure $(1,2,7,8,9,10,14,15,16$, 18,21).

Tension PNP is a very unusual cause of hemodynamic compromise of the blunt chest trauma patient. Clinical signs of cardiac tamponade, such as pulsus paradoxus, ta- chycardia, low - voltage electrocardiogram, increasing central venous pressure with decreasing cardiac output may indicate developing cardiac tamponade. However, the patients are often severely injured and other more common causes of hypotension (cardiac contusion, shock lung, hemorhagic shock, traumatic shock, tension pneumothorax, injury of the central nervous system) are considered first and may confuse the clinical picture (12).

It has also been suggested that some people may posses congenital pleural - pericardial connections $(8,11)$.

2) Alternatively the air from ruptured alveoli may penetrate along pulmonary venous perivascular and peribronchial sheats to the lung hilum and gain acces to the pericardium from here.

3) An additional possible mechanism is represented by the direct tracheobronchial - pericardial communication $(6,8,17)$.

Other authors have reported cases of PNP as a result of penetrating thoracic trauma $(4,5,19,20)$, infectious pericarditis with gas - forming organisms, and fistula formation between the pericardium and adjacent air - containing structures as the esophagus or stomach. Other etiologies include severe asthma, difficult labour, obstructive laryngitis, and therapeutic or diagnostic interventions such as proctocolectomy, laparoscopy, sternal bone marrow biopsy and tracheostomy. PNP may develop as a consequence of prolonged positive airway pressure (12).

Pneumopericardium is usually noted on admission or follow - up chest X-ray and CT examination. X-ray shows characteristic finding with air arround the cardiac silhouette (Fig. 1). The "small heart" sign may alert of the presence of tension pneumopericardium and impending cardiac tamponade $(12,13)$.

According to the basis of our experience the transesophageal echocardiography (TEE) is a method of choice for exclusion of air tamponade in these cases. We cannot see the air like pericardial effusion (air is not transmissive medium for ultrasound wawes) but with this method we can exactly measure the ejection fracture of the heart and see all echocardiographic symptoms of cardiac tamponade. On the basis of TEE we can select the patients for conservative or surgical treatment. We did not find this recommendation in any of the yet published papers.

As mentioned above PNP might be the sign of severe trauma which might lead to death, and therefore monitoring in an intensive care unit is mandatory. Tension pneumopericardium may occure also in patients with spontaneous breathing (14). Patients must be closely observed by means of clinical examinations, and serial of chest roentgenography, CT scans and eventually TEE must be done $(1,4$, $14,15,16,17,18)$.

Early bronchoscopy to exclude a tracheobronchial tear, which would require an immediate operative repair, is being recommended by some authors $(8,17,21)$.

On the basis of our experience we suggest, together with some others, that the presence of a pneumopericardium fol- 
lowing blunt chest trauma is not an absolute indication for surgery $(1,4,6,8,15,17)$. Pneumopericardium without the symptoms of cardiac tamponade is usually self limited and resolves spontaneously as we could observe in three our patients, too $(8,17)$.

Some authors advocate prophylactic pericardial decompression by means of the subxyphoid approach or percutaneous drainage to prevent potential gas tamponade, especially in patients who require mechanical ventilatory support $(7,14)$. But almost two thirds of these patients do not have this complication, and pneumopericardium was the cause of death in none of the fatal cases $(41,6 \%)(2,17)$.

In case of tension pneumopericardium Gould and Schurr (8) suggest, that percutaneous drains might not be sufficient to manage effectively the patient because the catheter may get plugged with pericardial sac debris. This should serve as temporary access to the sac for urgent decompression only. As the second step they recommend to perform permanent pericardial window in the operating suite. The window can be made either by an open subxyphoid approach, by open thoracotomy or by video assisted thoracoscopy with thoracic drain placement $(1,8)$.

\section{Conclusion}

Pneumopericardium is a very rare complication of blunt chest trauma and only several cases are reported in the literature. PNP without symptoms may be treated by observation. Tension PNP is best treated surgically by an open subxyphoid approach. The transesophageal echocardiography is a method of choice for excluding of air tamponade in these cases.

\section{References}

1. Baksaas ST, Fosse E, Pillgram-Larsen J. Traumatic pneumopericardium with cardiac tamponade. Tidsskr Nor Laegeforen 1992;112(16):2085-7.

2. Capizzi PJ, Martin M, Bannon MP. Tension pneumopericardium following blunt injury. J Trauma 1665;39:775-80.

3. Cimmiro CV. Some radio - diagnostic notes on pneumomediastinum, pneumothorax and pneumopericardium. Va Med 1967;94:205-213.

4. Demetriades D, Charalambides D, Pantanowitz D, et.al. Pneumopericardium following chest injuries. Arch Surg 1990;125(9):1187-9.
5. Demetriades D, Levy R, Hatzitheofilou C, et.al. Tension pneumopericardium following penetrating trauma: case report. J Trauma 1990:30(2):238-9.

6. Franklin WJ, Arora G, Ayres NA. Pneumopericardium and Pneumomediastinum in an Adolescent after Blunt Chest Trauma. Tex Heart inst J 2003;30(4): 338-339.

7. Gorecki PJ, Andrei VE, Schein M.Tension pneumopericardium in chest trauma. J Trauma 1999;46:954-6.

8. Gould JC, Schurr MA.Tension Pneumopericardium After Blunt Chest Trauma Ann Thorac Surg 2001;72:1728-1730.

9. Hudgens S, McGraw J, Craun M. Two cases of tension pneumopericardium following blunt chest injury. J Trauma 1991;31(10):1408-10.

10. Levin S, Maldonado i, Rehm C, et al. Cardiac tamponade without pericardial effusion after blunt chest trauma. Am Heart J 1996;13:198-200.

11. Lonský V, Štětina M, Habal P, et al. Congenital Absence of the Left Pericardium in Combination with Left Pulmonary Artery Hypoplasia, Right Aortic Arch and Secundum Atrial Septal Defect. The Thoracic and Cardiovascular Surgeon 1992; 40(3): $155-157$.

12. Mirvis SE. Imaging of Thoracic trauma. In: Turney SZ, Rodriguez A, Cowley RA, ed. Management of Cardiothoracic Trauma. Baltimore, Maryland: Williams \& Wilkins, 1990;27-93.

13. Mirvis SE, Indeck M, Schorr RM, et al. Posttraumatic tension pneumopericardium: the "small heart" sign. Radiology 1986;158(3):663-9.

14. Nakamura $T$, Tabuse $H$, Murao $Y$, et.al. 2 cases of the tension pneumopericardium following blunt chest trauma resulting in cardiac tamponade. Nippon Kyobu Geka Gakkai Zasshi.1994;42(8):1242-6.

15. Osinowo O, Adebo O. Levin S, Maldonado i, Rehm C, et al. Pneumopericardium following blunt chest injury: report of three cases. Injury1986;17(2):110-2.

16. Robinson MD, Markovchick VJ. Traumatic tension pneumopericardium: a case report and literature rewiew. J Emerg Med 1985;2(6):409-13.

17. Roth TC, Schmidt RA. Pneumopericardium after bunt chest trauma: A sign of severe injury? J Thorac Cardiovasc Surg 2002;124:630-631.

18. Shorr RM, Mirvis SE, indeck MC.Tension pneumopericardium in blunt chest trauma. J Trauma 1987;27(9):1078-82.

19. Spotnitz AJ, Kaufman JL. Tension pneumopericardium following penetrating chest injury. J Trauma 1987;27(7):806-8.

20. Tansel OD, Ozdemir H, Yamak B. Tension pneumopericardium in chest trauma with gunshot wound. Ulus Travma Derg 2003;9(4):304-6.

21. Wakaki N, Yamaguchi K, inouse S, et.al. Successful surgical treatment of patient with tension pneumopericardium after left hemothorax caused by multiple rib fractures. Kyobu Geka 1989;42(7):577-81.

Submitted February 2006.

Accepted May 2006.

MUDr. Vladimír Lonský, Ph.D., assoc. prof., FETCS,

University Hospital,

Department of Cardiac Surgery,

Sokolská 581,

50005 Hradec Králové,

Czech Republic.

e-mail: vlonsky@seznam.cz 